\title{
Distribución biogeográfica potencial del género Hippocampus Rafinesque 1810 (Actinopterygii: Syngnathiformes) en costas mexicanas
}

\section{Potential biogeographic distribution of the genus Hippocampus Rafinesque 1810 (Actinopterygii: Syngnathiformes) in Mexican coasts}

\author{
Jesús Alejandro Najera-Medellin ${ }^{1}$ y Antonio de la Mora-Covarrubias ${ }^{1}$
}

\begin{abstract}
RESUMEN
Los caballitos de mar (género Hippocampus) son peces cuyas poblaciones están amenazadas. Los mapas de distribución presentan información que puede utilizarse para la conservación de la biodiversidad, el correcto manejo y protección de los recursos naturales, y el desarrollo de planes de manejo, son la base para estudios posteriores de taxonomía, biogeografía, ecología y evolución. Con el fin de evaluar la eficiencia y el desempeño de diferentes métodos para el modelado de distribución potencial de las cuatro especies del género Hippocampus presentes en México, se recopilaron puntos georreferenciados de presencia de las mismas, desde 1970 hasta 2017, y se utilizaron cuatro modelos predictivos para determinar su distribución potencial. La evaluación de cada modelo se llevó a cabo con un análisis de curva ROC y un estadístico Kappa de Cohen. En MaxEnt se realizó un análisis Jacknife, para conocer las variables ambientales que tuvieron mayor importancia en la predicción del modelo. Los resultados muestran que $H$. erectus y $H$. zosterae tienen una amplia ordenación en las costas del Golfo de México; $H$. ingens muestra una marcada disposición a lo largo de los estados costeros del Océano Pacífico y $H$. reidi presenta una predicción de distribución más restringida en la zona del caribe mexicano. La batimetría, distancia a la costa y la concentración de clorofila son los factores con mayor influencia en el modelo predictivo de presencia de las cuatro especies.
\end{abstract}

Palabras clave: nicho, distribución potencial, modelos predictivos, Hippocampus, México

\section{ABSTRACT}

Seahorses (genus Hippocampus) are a group of threatened fish. Distribution maps present information for the conservation of biodiversity, correct management and protection of natural resources, and development of management plans. In addition, they are the basis for further studies

Instituto de Ciencias Biomédicas. Universidad Autónoma de Ciudad Juárez. Plutarco Elías Calles 1020, Fovissste Chamizal.32310 Ciudad Juárez, Chihuahua, México. al121474@alumnos.uacj.mx*, adelamor@uacj.mx 
in taxonomy, biogeography, ecology, and evolution. With the purpose of assessing the efficiency and performance of different methods for the modeling potential distribution of the four species of the genus Hippocampus in Mexico, georeferenced occurrence points were collected from 1970 to 2017, and four predictive models were used to determine their potential distribution. Each model was assessed with a ROC curve analysis and a Cohen's Kappa statistic. A Jacknife analysis was also conducted in the MaxEnt program to know the environmental variables that were more important in the model prediction. Results show that $H$. erectus and $H$. zosterae had a wide distribution in the coasts of the Gulf of Mexico; $H$. ingens shows a marked distribution along the coastal states of the Pacific Ocean while $H$. reidi has a more restricted distribution prediction in the Mexican Caribbean area. Bathymetry, distance to the coast, and chlorophyll concentration are the most determining factors in the probability of occurrence of the four species.

Keywords: Niche, potential distribution, predictive models, Hippocampus, Mexico

\section{INTRODUCCIÓN}

Los caballitos de mar constituyen un grupo de peces óseos distribuidos en los mares de regiones cálidas y templadas del mundo y sus 43 especies descritas están incluidas en el género Hippocampus (Lourie et al. 2016). Su peculiar constitución corporal y llamativa estrategia reproductiva son los aspectos más distintivos de todo el género (Lourie et al. 2004). Por su particular forma de vida, que incluye una muy reducida movilidad y elevada lealtad a su pareja, (Foster \& Vincent, 2004), aunado a que habitan ecosistemas marinos perturbados (pastos marinos, manglares, corales y estuarios), estos peces son particularmente vulnerables a la disminución en sus poblaciones (Bell et al. 2003). Se estima que aproximadamente 20 millones de ejemplares son comercializados anualmente, para su uso en acuariofilia como organismos de ornato, promoción turística como souvenirs, consumo como alimento gourmet y como ingrediente en la medicina tradicional china (Vincent, 1996; McPherson \& Vincent, 2004; Rosa et al. 2007; Vincent et al. 2011). Por otro lado, los caballitos de mar se han considerado "especies bandera" y sus datos de distribución pueden ser utilizados para delimitar áreas naturales protegidas (Luzzato \& Pujol, 2014; Zhang \& Vincent, 2017).

Tradicionalmente, los inventarios de campo eran los métodos más sencillos para establecer la distribución de las especies, sin embargo, demandaban alta inversión económica y de tiempo. Hoy, los Sistemas de Información Geográfica (SIG) han permitido predecir el nicho ecológico potencial de las especies, utilizando datos de presencia/ausencia y algoritmos desarrollados para este 
propósito. Los mapas de distribución presentan información valiosa para la conservación de la biodiversidad, y son una herramienta útil para el correcto manejo y protección de los recursos naturales, para el desarrollo de planes de manejo y pueden ser de gran ayuda para estudios posteriores de taxonomía, biogeografía, ecología y evolución (Pimm et al. 2014). El mapeo y modelaje potencial de especies marinas, es un reto particular que han sido poco documentadas (Guisan \& Thuiller, 2005; Franklin, 2010). En México, se reporta la presencia de cuatro especies de caballitos de mar con categoría de protección especial (Pr) dentro de la NOM-059-SEMARNAT-2010: $H$. ingens en la costa del Océano Pacífico; $H$. erectus, $H$. zosterae y $H$. reidi en el Golfo de México y Mar Caribe (Bruckner et al. 2005). Generar conocimiento en su distribución en el vasto territorio marino del país podría ayudar a la aplicación de medidas para su protección o aprovechamiento sustentable.

Esta investigación ofrece una metodología que integra los algoritmos de modelado de nicho ecológico, que permite estimar la distribución potencial de las cuatro especies del género Hippocampus presentes en México, lo que puede ser de utilidad para identificar zonas donde habiten poblaciones de caballitos de mar.

\section{MATERIALES Y MÉTODOS}

Área de estudio. México se ubica en la latitud Norte, entre las coordenadas extremas $14^{\circ} 32^{\prime} 27^{\prime}$ ' y $32^{\circ}$ 43' 06" y en la longitud Oeste entre las coordenadas extremas $86^{\circ} 42^{\prime} 36^{\prime \prime}$ y $118^{\circ} 22^{\prime}$ 00', (Fig. 1). En su parte media lo atraviesa la línea del Trópico de Cáncer (INEGI, 2009). El territorio terrestre y para homogenizar la información geográfica todas las coordenadas fueron transformadas al sistema Universal Transversal de Mercator (UTM). Lo anterior le confiere a México una extraordinaria riqueza faunística, florística y ficológica (CONABIO, 2008).

Puntos de presencia. Se generó una base de datos para cada una de las cuatro especies a evaluar (H. erectus, $H$. ingens, $H$. reidi y $H$. zosterae) utilizando la información obtenida de la colección científica ictiológica de la Universidad Nacional Autónoma de México, las publicaciones científicas y las bases de datos electrónicas de FishNet, Fishbase, Vertnet y GBFI (Global Biodiversity Information Facility, 2017). Las variables por considerar fueron: género, especie, localidad de registro, profundidad, longitud y latitud. Para homogenizar la información geográfica todas las coordenadas fueron transformadas al sistema Universal Transversal de Mercator (UTM). 


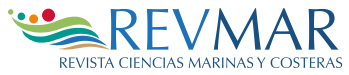

$a$
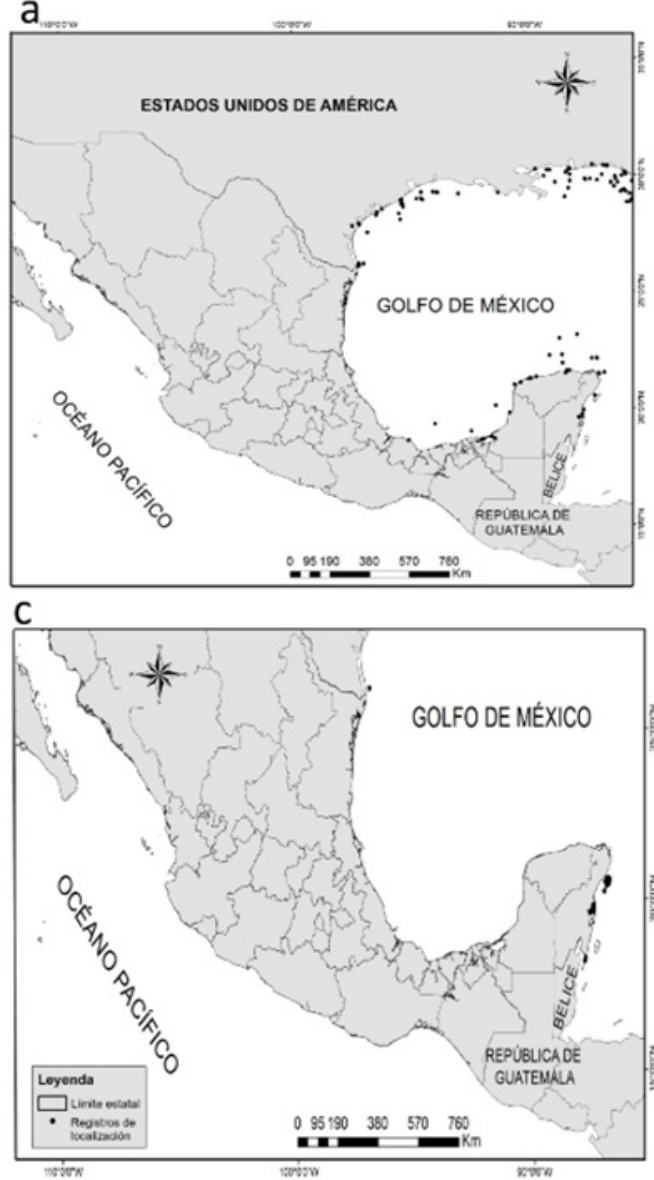

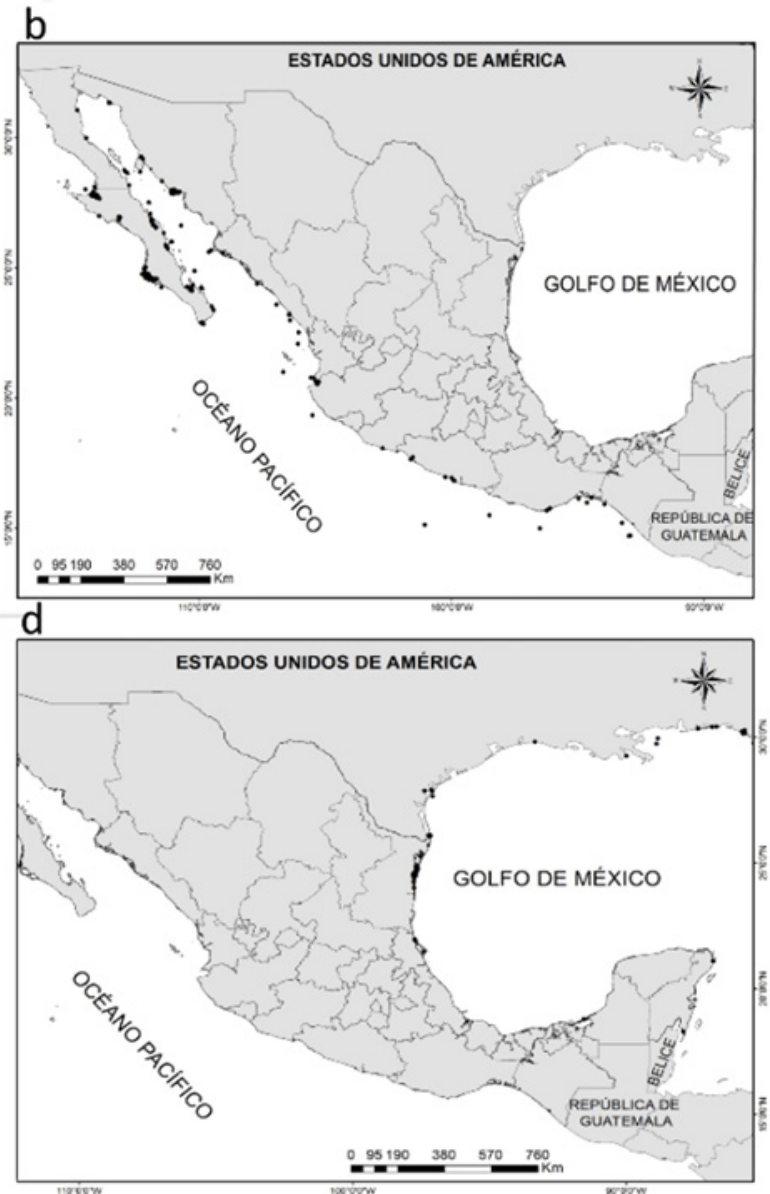

Fig. 1. Registros de localización del género Hippocampus en las costas de México: A) H. erectus; B) $H$. ingens; C) $H$. reidi y D) $H$. zosterae Fig. 1. Location records of the genus Hippocampus in the coasts of Mexico: A) $H$. erectus; B) $H$. ingens; C) $H$. reidi, and D) $H$. zosterae

Variables ambientales. Las capas de datos ambientales (Cuadro 1) se obtuvieron del sitio electrónico del Global Marine Environment Dataset (http://gmed.auckland.ac.nz) versión 1.0 , con una característica de 5 arc-min lat-long (aproximadamente $9.2 \mathrm{~km}$ por cuadrícula cerca del ecuador). Las capas se ingresaron al software ArcGis 10.3 y se recortaron utilizando como máscara la capa de las ecorregiones de México (Toledo \& Ordoñez, 2009). 
Cuadro 1. Variables ambientales utilizadas para predecir la distribución potencial de las cuatro especies del género Hippocampus en México

Table 1. Environmental variables used to predict the potential distribution of the four species of the genus Hippocampus in Mexico

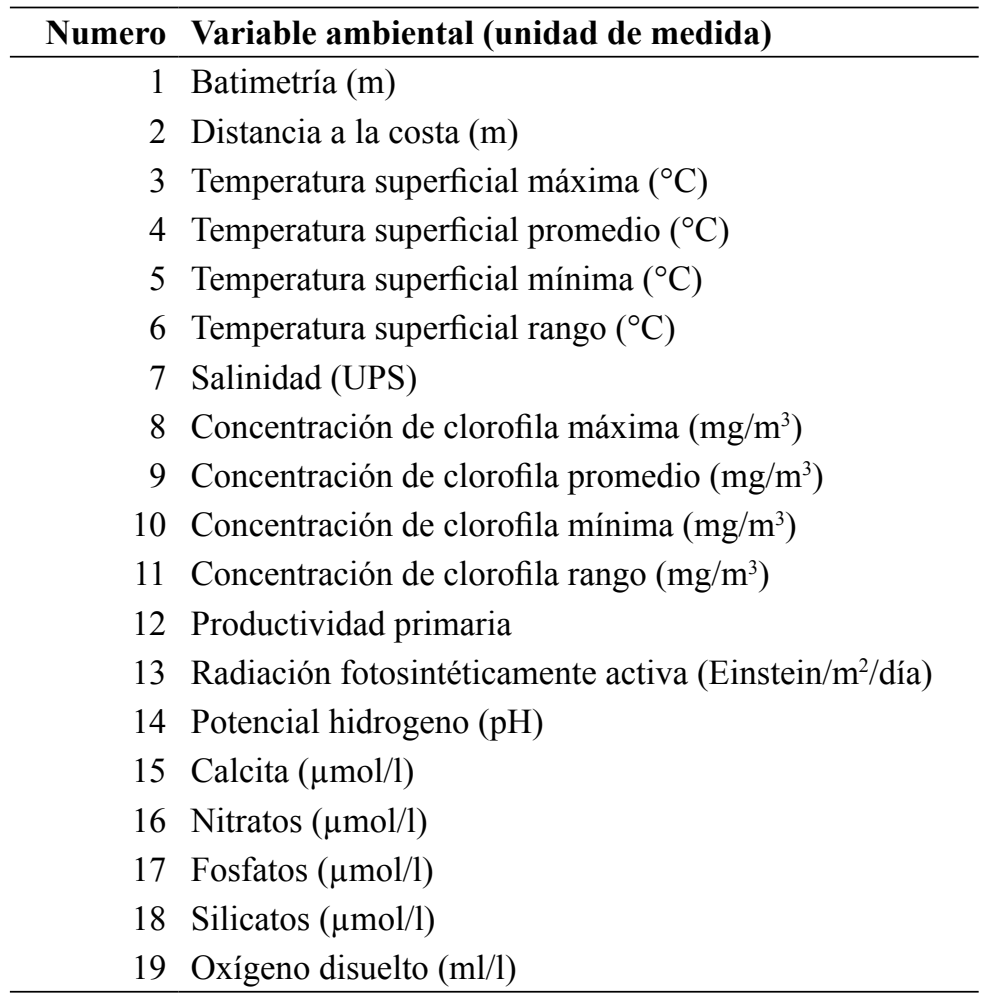

Ejecución de los modelos. climáticos y los relaciona con las Los modelos BIOCLIM (Perfil ocurrencias (registros) de la especie Bioclimático), DOMAIN (Métrica de Gower), GARP (Genetic Algorith for Rule Set Production) y MaxEnt (Máxima Entropía) fueron utilizados para predecir la distribución potencial del género Hippocampus en México. Estos modelos realizan un contraste entre los factores ecológicos $\mathrm{y}$ (Guisan \& Thuiller, 2005).

Los datos de presencia de las cuatro especies fueron formateados para que el programa DIVAGIS 5.2 leyera los archivos con las siguientes características: especie (género y epíteto) y coordenadas geográficas (latitud-longitud) en WGS-1984. Las 19 variables 
bioclimáticas seleccionadas fueron apiladas virtualmente y se procedió a ejecutar el algoritmo BIOCLIM y DOMAIN en el software para obtener el mapa. Se utilizó el dominio extenso $(0-100 \%)$ (Villaseñor \& Tellez-Valdés, 2004; García, 2006) para considerar la probabilidad de presencia en ambos modelos. Se aplicaron los criterios de cinco, cuatro, tres y dos cuartiles para considerar la presencia con una probabilidad $\geq 0.5$ en las opciones de etiqueta de ArcGis ver. 10.3.

El algoritmo GARP fue ejecutado en el software de uso libre OpenModeller Desktop 1.1.0. Se realizaron 100 iteraciones o combinaciones entre variables ambientales y registros de presencia de cada especie evaluada. Cada iteración produce un mapa de presencia/ausencia. Estos se compilan para obtener una predicción consenso para cada especie.

El formato de salida para el algoritmo MaxEnt (ver. 3.3.3) fue el logístico con un análisis automatizado de datos. El conjunto de datos fue divido aleatoriamente en $25 \%$ para su evaluación, mientras que el $75 \%$ restante fue utilizado para el entrenamiento del modelo (Echarri et al. 2009). El criterio utilizado para denotar la presencia fue el valor arrojado por el límite logístico "10 percentile training presence", el cual varió en cada especie: 0.350 para $H$. erectus; 0.219 en $H$. ingens; 0.212 en $\mathrm{H}$. reidi y 0.256 para H. zosterae.
Evaluación de los modelos. El desempeño fue evaluado utilizando los valores del área bajo la curva ROC/AUC en el programa MaxEnt, y la validación se realizó con el índice Kappa de Cohen, este se obtuvo del software de DIVAGIS para todas las especies en todos los algoritmos. En general, se consideraron valores mayores a 0.80 como predicciones satisfactorias (Araujo \& Guisan, 2006; García, 2006).

Selección de las variables de importancia. Se realizó una prueba Jacknife en el programa MaxEnt 3.3.3. Este procedimiento establece la contribución de cada variable al modelo (Phillips et al. 2006). Los mapas finales se maquetaron en el programa ArcGIS 10.3, se utilizó en todos los mapas el datum geodésico WGS84 y una escala de 1:8.000.000.

\section{RESULTADOS}

Se recolectaron 729 registros para el género Hippocampus en México del periodo del año 1970 al año 2017. H. erectus presentó el mayor número de registros con 304 , seguido de $H$. ingens con $249 ; H$. zoosterae con 126 y 50 para $H$. reidi (Fig. 1).

Predicción de la distribución potencial para $H$. erectus. El resultado de los cuatro modelos (Fig. 2) destaca una probabilidad alta de presencia 
de $H$. erectus en las costas del Golfo de México, mostrando viabilidad ambiental para sostener poblaciones en litorales y mares de los estados de Tamaulipas, Veracruz, Tabasco, Campeche, Yucatán y Quintana Roo. En el
Océano Pacífico los modelos de BIOCLIM y GARP destacan predicción de probabilidad en los estados de Nayarit, Jalisco, Colima y Michoacán, en los cuales no se ha reportado avistamiento de esta especie.
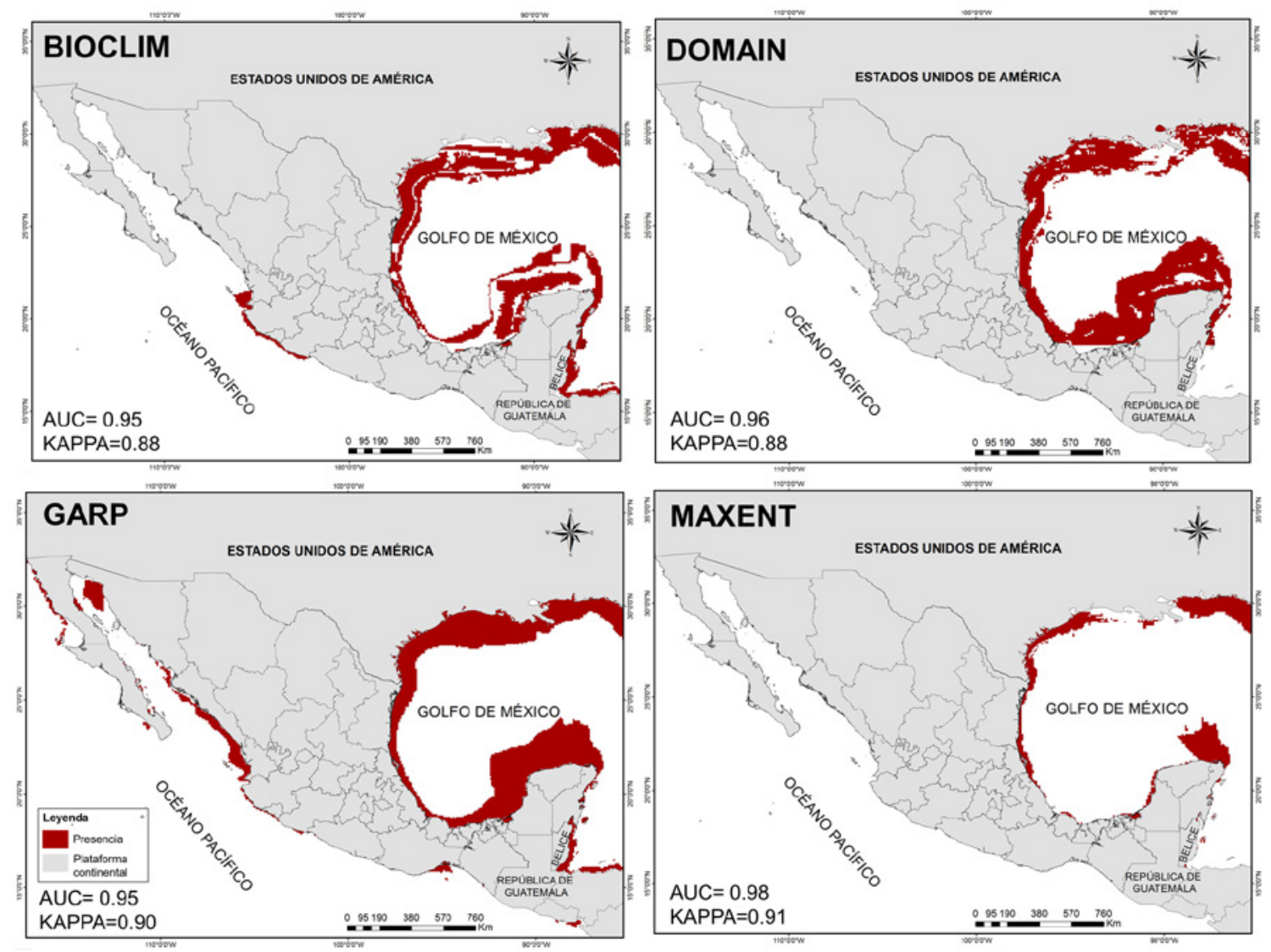

Fig. 2. Modelos de distribución potencial de la especie Hippocampus erectus en México. En la parte inferior izquierda se muestran los valores AUC/KAPPA para cada uno de los algoritmos

Fig. 2. Potential distribution models of the species Hippocampus erectus in Mexico. AUC/KAPPA values for each of the algorithms are shown in the lower left corner 
Predicción de la distribución Baja California (a excepción del algopotencial para $\boldsymbol{H}$. ingens. El resul- ritmo GARP). Destaca además zonas tado de los modelos prueba (Fig. 3) de presencia potencial en los MDE de destaca una probabilidad alta de $H$. in- BIOCLIM y GARP para los estados de gens en todos los estados costeros del Océano Pacífico, se excluyó el área del delta del Río Colorado en Sonora y Veracruz, Tabasco y Campeche, sitios donde no se ha avistado la especie.
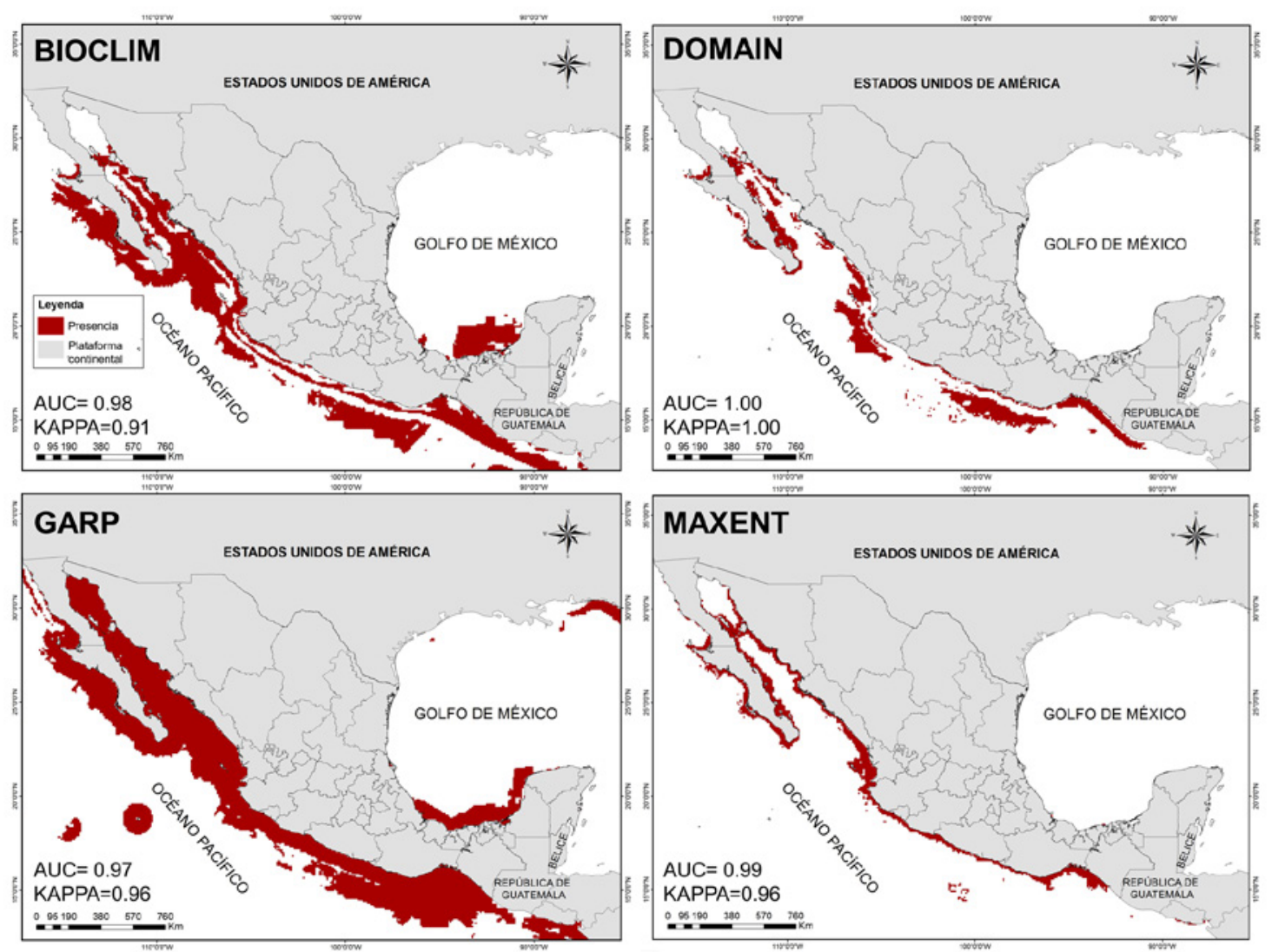

Fig. 3. Modelos de distribución potencial de la especie Hippocampus ingens en México. En la parte inferior izquierda se muestran los valores AUC/KAPPA para cada uno de los algoritmos

Fig. 3. Potential distribution models of the species Hippocampus ingens in Mexico. AUC/KAPPA values for each of the algorithms are shown in the lower left corner 
Predicción de la distribución de Tamaulipas, Veracruz, Yucatán y potencial para $\boldsymbol{H}$. reidi. El resultado Quintana Roo, aunque la distribución de los modelos evaluados (Fig. 4) des- es difusa y en parches, apreciándose taca una probabilidad alta de presencia mejor en la parte norte de Tabasco y en para $H$. reidi en el litoral del Golfo de la zona caribeña (Quintana Roo), preMéxico, mostrando viabilidad ambien- sentando resultados similares al modetal para sostener poblaciones en las lo DOMAIN. No se aprecia predicción costas y aguas marinas de los estados en el Océano Pacífico.

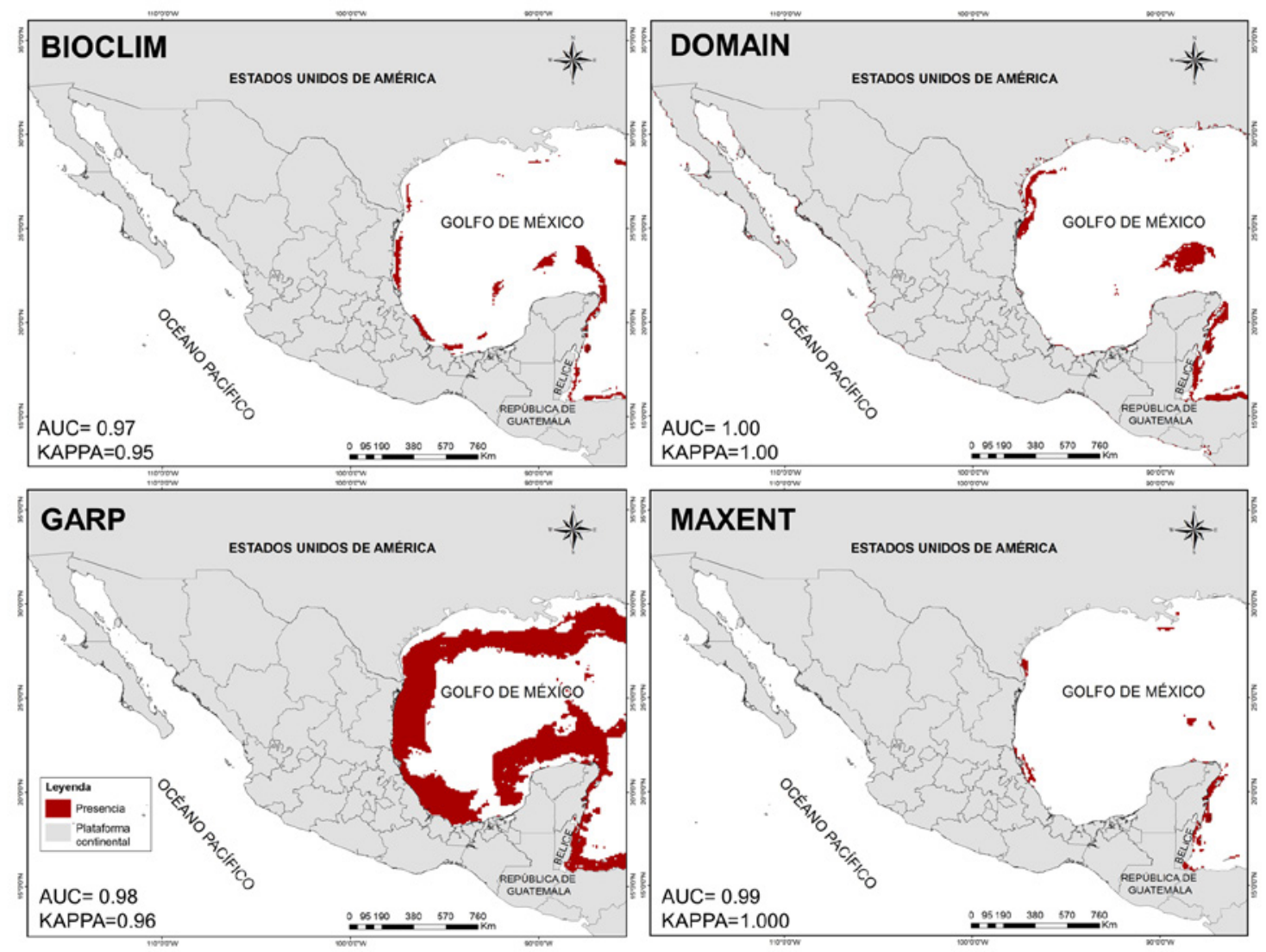

Fig. 4. Modelos de distribución potencial de la especie Hippocampus reidi in México. En la parte inferior izquierda se muestran los valores AUC/KAPPA para cada uno de los algoritmos

Fig. 4. Potential distribution models of the species Hippocampus reidi in Mexico. AUC/KAPPA values for each of the algorithms are shown in the lower left corner 
Predicción de la distribución en aguas costeras y mares de los espotencial para H. zosterae. El resulta- tados de Tamaulipas, Veracruz, Tabasdo de los modelos evaluados (Fig. 5) co, Campeche, Yucatán y Quintana destaca una probabilidad alta de pre- Roo. Se muestran pequeños parches sencia para $H$. zosterae en el litoral del de áreas con probabilidad en todos los Golfo de México, mostrando viabilidad estados costeros del Océano Pacífico. ambiental para sostener poblaciones
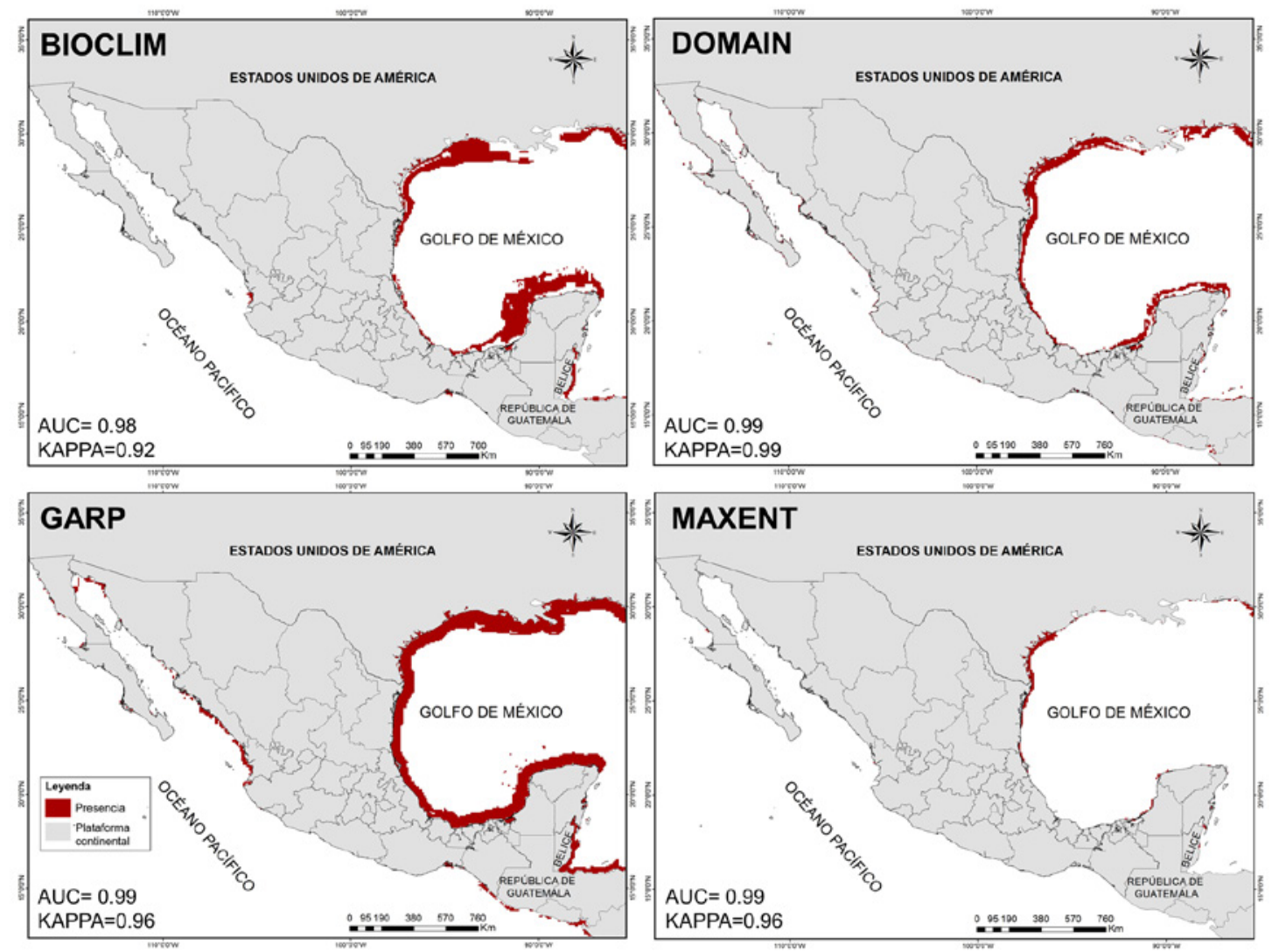

Fig. 5. Modelos de distribución potencial de la especie Hippocampus zosterae en México. En la parte inferior izquierda se muestran los valores AUC/KAPPA para cada uno de los algoritmos

Fig. 5. Potential distribution models of the species Hippocampus zosterae in Mexico. AUC/KAPPA values for each of the algorithms are shown in the lower left corner 
El cuadro 2 muestra las tres variables explicativas (climáticas y ambientales) de mayor peso para el modelo predictivo, las cuales fueron obtenidas del análisis Jacknife. La batimetría pudiera considerarse como la de mayor contribución exceptuando a $H$. ingens.

Los valores estimados de AUC y Kappa para cada uno de los modelos evaluados muestran de manera general un desempeño satisfactorio en todos los algoritmos (Fig. 2, Fig. 3, Fig. 4 y Fig. 5).

\section{DISCUSIÓN}

El modelado de nicho ecológico (MDE) es un enfoque ampliamente utilizado en estudios con sistemas terrestres, ya que permite la gestión, la conservación y la planificación de las poblaciones silvestres y cinegéticas (Anderson et al. 2002; Chefauri et al.
2005), no obstante, ha sido poco utilizado en los sistemas acuáticos (Wiley et al. 2003; McNyset, 2005). Un desafío es que la amplia variabilidad espacio-temporal que caracteriza a los océanos puede dificultar el modelado de espacio ecológico (Valavanis et al. 2008; Franklin, 2010). Un segundo obstáculo es la disponibilidad restringida de variables ambientales y bioclimáticas de ecosistemas marinos, ya que no suelen encontrarse con acceso libre, resolución apropiada, escala espacial específica, y aquellos que se encuentran disponibles con frecuencia tienen ausencias importantes de las regiones costeras (Kaschner et al. 2006), lo que hace que el ensamblaje de un conjunto de datos sea uno de los aspectos más lentos para la predicción (Hijmans et al. 2005).

LosalgoritmosdeMDErequieren registros de presencia de alta calidad e información ambiental, para inferir sus

Cuadro 2. Variables explicativas de mayor importancia en la predicción del modelo

Table 2. Most important explanatory variables in the model prediction

\begin{tabular}{|c|c|c|c|}
\hline \multirow{2}{*}{ Especie } & \multicolumn{3}{|c|}{ Variable explicativa (\% de contribución al modelo) } \\
\hline & 1 & 2 & 3 \\
\hline $\begin{array}{l}\text { Hippocampus } \\
\text { erectus }\end{array}$ & Batimetría (65.5) & Rango de temperatura (9.1) & $\mathrm{Ph}(9.0)$ \\
\hline $\begin{array}{l}\text { Hippocampus } \\
\text { ingens }\end{array}$ & $\begin{array}{l}\text { Distancia a la costa } \\
(43.6)\end{array}$ & $\begin{array}{l}\text { Concentración de clorofila } \\
\text { máxima (16.7) }\end{array}$ & $\begin{array}{l}\text { Productividad primaria } \\
\text { (11.7) }\end{array}$ \\
\hline Hippocampus reidi & Batimetría (40.7) & Salinidad (23) & Fosfatos (12.4) \\
\hline $\begin{array}{l}\text { Hippocampus } \\
\text { zosterae }\end{array}$ & Batimetría (63) & $\begin{array}{l}\text { Concentración de clorofila } \\
\text { máxima (13.2) }\end{array}$ & Distancia a la costa (7) \\
\hline
\end{tabular}


preferencias macroecológicas (Elith \& Leathwick, 2009), lo cual puede resultar aún más difícil en especies que son pobremente registradas, como es el caso del género Hippocampus (Zhang \& Vincent, 2017). Este problema se puede compensar integrando múltiples bases de datos para homogeneizar y complementar los registros de presencia.

Es común que en este tipo de estudios ocurran sobreestimaciones, cuyas causas pueden ser desde algorítmicas, metodológicas y hasta conceptuales. Algunas investigaciones comparativas entre MaxEnt y GARP (Elith, 2006; Pearson et al. 2006) muestran que clasifican erróneamente la presencia-ausencia de la especie y por ende sobreestiman la predicción, sin embargo, estos solo se enfocaron en la capacidad de interpolación (predicción de presencia en zonas con puntos de muestreo) de los modelos. Aquellos donde la extrapolación de los datos (predicción en zonas donde no existió muestreo) fue el objetivo principal, MaxEnt ha probado ser eficiente (Peterson, 2001).

Otro factor importante es que los modelos de predicción utilizan las condiciones ambientales en las que los organismos se pueden desarrollar exitosamente, pero excluyen factores geográficos, ecológico e históricos que puedan dificultar que aprovechen totalmente la extensión de ese nicho ecológico potencial, por ende, que una espe- cie no se encuentre en un determinado lugar no es sinónimo de una ausencia en su nicho (Soberon \& Peterson, 2005). Esto es determinante en los modelos obtenidos, ya que en otros (GARP y Bioclim) apareció probabilidad de presencia en zonas marítimas, donde no se ha reportado anteriormente.

Las cuatro especies de caballitos de mar residentes, al igual que algunas otras de las que componen al género Hippocampus, se caracterizan por su alta fidelidad al sitio y bajo rango de dispersión (Lourie et al. 2016), lo que indica un gran aislamiento entre poblaciones que se ven afectadas por las condiciones físicas, químicas, nutrimentales y biológicas de la zona donde se establecen, sin embargo hay otras lugares con parámetros físicos y químicos similares y en donde potencialmente pueden establecerse.

El presente trabajo no contempla metadatos de la preferencia de hábitat de las especies evaluadas, ya que no se encontraban para una escala nacional. Si bien se ha demostrado que el análisis de la distribución potencial es más preciso al emplear variables que contemplen la proximidad del hábitat (Zhang \& Vincent, 2018), los resultados obtenidos son muy similares a los reportados por las autoras anteriormente citadas, además se optó por omitir dichos factores, ya que son empleados en su mayoría para animales con amplio rango de movilidad y a escala regional o local (p. ej. peces arrecifales) 
(Shelton et al. 2014), muchas de las áreas con probabilidad de ocurrencia en nuestros modelos se encuentran en lugares de fondo abierto (arenosos y fangosos), así como zonas artificiales (redes de natación en puertos y granjas acuícolas). Alentamos futuros estudios de MDE sobre hipocampos en México y el resto de Latinoamérica a escala local y regional para incluir estos datos de hábitat, si están disponibles.

Los patrones de distribución predichos para las cuatro especies en México son consistentes con sus contrapartes en otras regiones. H. erectus es una especie encontrada frecuentemente en zonas profundas con abundante vegetación (generalmente, bosques de algas) (Teixeira y Musick, 2001), lo que coincide con el patrón de distribución obtenido por MaxEnt, BIOCLIM y DOMAIN. De acuerdo con Basher et al. (2014), las zonas que presentan mayor profundidad en el Golfo de México son Yucatán, Quintana Roo, Tabasco y Veracruz, lo cual se puede corroborar en las zonas de predicción, las cuales son similares en todos los algoritmos utilizados. En cuanto la predicción de presencia en las costas del Pacífico, esto pudo haberse visto influenciado en el modelo por las características similares del rango de temperatura en la zona costera de Nayarit, Jalisco Colima y Michoacán, la cual es similar a la presentada en los estados del Golfo de México.

Como peces ectodérmicos, se espera que los hipocampos requieran temperaturas adecuadas del agua para mantener su metabolismo y reproducirse (Beitinger \& Fitzpatrick, 1979). $H$. ingens es una especie que se caracteriza por tener preferencia por aguas con temperaturas templadas $\left(14-21^{\circ} \mathrm{C}\right)$ (Lourie et al. 2004), la cual se ha diversificado y establecido únicamente en las costas americanas del Océano Pacífico. En el caso de H. reidi se vio más restringido a las aguas cálidas y poco profundas del caribe mexicano. Dias y Rosa (2003) mencionan que esta especie muestra fuerte atracción hacia varios de los microhábitats presentes en muelles, como bosques de macroalgas, esponjas, cnidarios y tunicados, los cuales son comunes en algunas zonas de Quintana Roo (Parque Nacional Arrecifes de Cozumel y Reserva de Sian Ka'an) y Tamaulipas (Laguna Madre, en la cual se han registrado las tres especies de caballitos de mar residentes en el Golfo de México) (CONABIO, 2009).

Finalmente, $H$. zosterae mostró una distribución similar a la de $H$. erectus, aunque a diferencia de esta última, tiene un rango de dispersión más amplio, ya que se ha reportado en lechos de algas marinas en verano, y en invierno tiende a migrar a aguas poco profundas encontrándose asociado a comunidades de pastos marinos (Lourie et al. 2004; Lourie, 2016).

Sobre las variables de mayor importancia en los modelos predictivos cada especie mostró perfiles diferentes. 
Para H. erectus fueron la batimetría, el rango de temperatura y el $\mathrm{pH}$. Para esta especie la profundidad máxima reportada es de $73 \mathrm{~m}$ (Vari, 1982), y el mayor número de observaciones se encuentra asociado a ecosistemas de bajas profundidades (menores a dos metros) como comunidades de algas marinas, esponjas, sargazo flotante y manglar sumergido (Pastor-Gutiérrez et al. 2011) que se encuentran por lo general en aguas con profundidad mayor a $10 \mathrm{~m}$, aunque también, se ha reportado en áreas no tan profundas como bahías, playas y marismas (Lourie, 2016).

En $H$. ingens se determinó que la distancia a la costa, la concentración máxima de clorofila y la productividad primaria son las variables determinantes en la predicción del modelo, lo cual es consistente con la ecología de la especie, ya que normalmente, se encuentra en aguas poco profundas de 1-20 m cercanas a la costa (Lourie et al. 2004); ya sea entre gorgonias o coral negro (Humann, 1993), así como en pastos marinos (Groves \& Lavenberg, 1997).

De acuerdo con Basher et al. (2014), la productividad primaria y la concentración de clorofila están directamente relacionados, y generalmente, la mayor productividad se da en mares fríos, lo cual explica la preferencia de mares templados, por esta especie.

Para $H$. reidi la salinidad, la batimetría y los fosfatos son los parámetros que mayor ganancia tienen dentro del modelo. Esta especie generalmente, se encuentra a 2-55 $\mathrm{m}$ de profundidad (Vari, 1982; Pastor-Gutiérrez et al. 2011) en raíces de manglar, algas marinas, campos de ostras, cnidarios, esponjas, tunicados, estructuras artificiales en estuarios y gorgonias (Rosa et al. 2007; Pastor-Gutiérrez et al. 2011). Por otra parte, el rango de fosfatos tolerable registrado es de $0.12 \mu \mathrm{mol} / \mathrm{L}$ a 1.2 $\mu \mathrm{mol} / \mathrm{L}$ (Lourie et al. 2004) y de acuerdo con Basher et al. (2014), la zona del Golfo de México y en general el Océano Atlántico presenta un rango bajo de concentración de fosfatos (0-0.26 $\mu \mathrm{mol} / \mathrm{L})$, aunque este parámetro puede verse fuertemente influido por actividades agrícolas y del sector terciario.

Finalmente, para la especie $H$. zosterae, la batimetría, la concentración de clorofila máxima y la distancia a la costa fueron las variables con mayor aportación a la predicción. Debido a que esta especie tiende a migrar a aguas poco profundas en temporadas donde la temperatura desciende es común encontrarla asociada a comunidades de praderas de pastos marinos (Lourie et al. 2004; Lourie, 2016), los cuales por lo general se encuentran en aguas con una profundidad no mayor a los $10 \mathrm{~m}$ y cercanos a la costa, así mismo son productores primarios de una gran cantidad de materia orgánica (Brigitta et al. 2010).

El hábitat del caballito de mar enano se caracteriza por arenales poco profundos, cercanos a la costa, que varían de uno a cuatro metros de pro- 
fundidad, típicamente dominados por praderas de algas marinas y macroalgas (Fedrizzi et al. 2015).

Acerca de la evaluación de los modelos, Thullier et al. (2009) mencionan que cifras por arriba de 0.9 de AUC y de Kappa dan cuenta de predicciones con alta precisión y de desempeño global satisfactorio, demostrando que se ajustan a la muestra de evaluación, más allá de lo esperado por azar. Prácticamente, en todos los MDE obtenidos los resultados fueron mayores a 0.9 para AUC y muy cercanos a 1 para Kappa. Sin embargo, se debe tener en cuenta que el valor del AUC depende del número de presencias y del tamaño total del área de estudio (Lobo et al. 2008), por lo que los resultados deberían tomarse con cautela.

\section{CONCLUSIÓN}

A pesar de las limitaciones para el modelado de especies marinas, los resultados encontrados nos permiten concluir que para el caso del género Hippocampus, el algoritmo que ofrece mayor confianza para la toma de decisiones relacionadas a la predicción de distribución espacial de nicho es MaxEnt, ya que presentó los mejores resultados en la evaluación AUC y Kappa. Se sugiere llevar a cabo estudios de MDE a una escala local y regional al utilizar metadatos que contemplen el hábitat, así como un trabajo de campo dirigido que ayude a corroborar los hallazgos.

\section{BIBLIOGRAFÍA}

Anderson, R., Gómez-Laverde, M. \& Peterson, A. (2002). Geographical distributions of spiny pocket mice in South America: insights from predictive models. Glob. Ecol. Biog., 11(2), 131141. https://doi.org/10.1046/j.1466822X.2002.00275.X

Araújo, M. B. \& Guisan, A. (2006). Five (or so) challenges for species distribution modeling. J. Biogeogr., 33(10), 16771688. https://doi.org/10.1111/j.13652699.2006.01584.x

Basher, Z., Bowden, D. A. \& Costello, M. J. (2014). Global Marine Environment Datasets (GMED). World Wide Web electronic publication. Version 1.0 (Rev.01.2014). Recuperado en agosto 14, 2017, disponible en http://gmed. auckland.ac.nz

Beitinger, T. L. \& Fitzpatrick, L. C. (1979). Physiological and ecological correlates of preferred temperature in fish. Integr. Comp. Biol., 19(1), 319-329. https:// doi.org/10.1093/icb/19.1.319

Bell, E. M., Lockyear, J. F., McPherson, J. M., Marsden, A. D. \& Vincent, A. C. J. (2003). First field studies of an Endangered South African seahorse, Hippocampus capensis. Environ Biol.

Fish., 67(1), 35-46. https://doi.org/10.1023/A:1024440717162

Brigitta, I., Van Tussenbroek, M., Barba Santos, G., Gonzalo, J., Wong R., Kornelis van Dijk, J. \& Waycott, M. (2010). Guía de los pastos marinos tropicales del Atlántico oeste / A guide to the tropical seagrasses of the Western Atlantic. México: Univ. Nac. Aut. de Méx.

Bruckner, A. W., Field, J. D. \& Daves, N. (Eds.). (2005). The Proceedings of 
the International Workshop on CITES Implementation for Seahorse Conservation and Trade. Silver Spring, Maryland, EE. UU.: National Oceanic and Atmospheric Administration.

Chefaoui, R. M., Hortal, J. \& Lobo, M. (2005). Potential distribution modeling, niche characterization and conservation status assessment using GIS tools: a case study of Iberian Copris species. Biol. Conserv., 122(2), 327-338. https://doi. org/10.1016/j.biocon.2004.08.005

CONABIO. Comisión Nacional para el Conocimiento y Uso de la Biodiversidad. (2008). Capital natural de México, vol. I: Conocimiento actual de la biodiversidad. México: Comisión Nacional para el Conocimiento y Uso de la Biodiversidad.

CONABIO. Comisión Nacional para el Conocimiento y Uso de la Biodiversidad. (2009). Capital natural de México, vol. II: Estado de conservación y tendencias de cambio. México: Comisión Nacional para el Conocimiento y Uso de la Biodiversidad.

Dias, T. L. P. \& Rosa, I. L. (2003). Habitat preferences of a seahorse species, Hippocampus reidi (Teleostei: Syngnathidae) in Brazil. Aq. J. Ichth. Aq. Biol., 6(4), 165-176.

Echarri, F., Tambussi, C. \& Hospitaleche, C. A. (2009). Predicting the distribution of the crested tinamous. Eudromia spp. (Aves, Tinamiformes). J. Ornithol., 150, 75-84. https://doi.org/10.1007/ s10336-008-0319-5

Elith, J. \& Leathwick, J. R. (2009). Species distribution models: ecological explanation and prediction across space and time. Annu. Rev. Ecol. Evol. S., 40, 677-697. https://doi.org/10.1146/annurev.ecolsys. 110308.120159
Elith, J., Graham, C. H., Anderson, P. R., Dudík, M., Ferrier, S., Guisan, A., Zimmermann, N. E. (2006). Novel methods improve prediction of species distributions from occurrence data. Ecography, 29(2), 129-151. https://doi. org/10.1111/j.2006.0906-7590.04596.x

Fedrizzi, N., Stiassny, M. L. J., Boehm, J. T., Dougherty, E. R., Amato, G. \& Mendez, M. (2015). Population Genetic Structure of the Dwarf Seahorse (Hippocampus zosterae) in Florida. PLOS ONE, 10(7), e0132308. https://doi. org/10.1371/journal.pone.0132308

Foster, S. J. \& Vincent, A. C. J. (2004). The life history and ecology of seahorses: implications for conservation and management. J. Fish Biol., 65(1), 1-61. https://doi.org/10.1111/j.00221112.2004.00429.x

Franklin, J. (2010). Mapping Species Distributions: Spatial Inference and Prediction. Cambridge, UK.: Cambridge University Press. https://doi.org/10.1017/ CBO9780511810602

García, J. R. (2006). Multi-scale assessment of the potential distribution of two herptofaunal species. Tesis de maestría no publicada. Wageningen University, Países Bajos.

Global Biodiversity Information Facility. (2017). GBIF Home Page. Recuperado en abril 11, 2017, disponible en https:// www.gbif.org

Groves, J. S. \& Lavenberg, R. J. (1997). The Fishes of the Galapagos Islands. Stanford, EE. UU.: Stanford University Press.

Guisan, A. \& Thuiller, W. (2005). Predicting species distribution: offering more than simple habitat models. Ecol. Letters, 8(9), 993-1009. https://doi. org/10.1111/j.1461-0248.2005.00792.x 
Hijmans, R. J., Cameron, S. E., Parra, J. L., Jones, P. G. \& Jarvis, A. (2005). Very high resolution interpolated climate surfaces for global land areas. Int. J. Clim., 25(15), 1965-1978. https://doi. org/10.1002/joc. 1276

Humann, P. (1993). Reef fish identification: Galapagos. Florida, EE. UU.: New World Publications.

INEGI. Instituto Nacional de Estadística y Geografía. (2009). Aspectos generales del territorio mexicano. Recuperado en enero 2 2016, disponible enhttp:// www.inegi.org.mx/inegi/default.as$\mathrm{px}$ ? $=$ geo\&c $=909$.

Kaschner, K., Watson, R., Trites, A. W. \& Pauly, D. (2006). Mapping worldwide distributions of marine mammal species using a relative environmental suitability (RES) model. Mar. Ecol.

Prog. Ser., 316, 285-310. https://doi. org/10.3354/meps316285

Lobo, J. M., Jiménez-Valverde, A. \& Real, R. (2008). AUC: a misleading measure of the performance of predictive distribution models. Global Ecol. Biogeogr., 17(2), 145-151. https://doi.or$\mathrm{g} / 10.1111 / \mathrm{j} .1466-8238.2007 .00358 . \mathrm{x}$

Lourie, S. A. (2016). Seahorses-A life size Guide to every species. Vernmont, EE. UU.: Ivy Press. https://doi.org/10.7208/ chicago/9780226338552.001.0001

Lourie, S. A., Foster, S. J., Cooper, E. W. T. \& Vincent, A. C. J. (2004). A guide to the identification of seahorses. Project Seahorse and Traffic North America. Washington D. C. EE. UU.: University of British Columbia \& World Wildlife Fund.

Lourie, S. A., Pollom, R. A. \& Foster, S. J. (2016). A global revision of the Seahorses Hippocampus Rafinesque 1810 (Actinopterygii: Syn- gnathiformes): taxonomy and biogeography with recommendations for further research. Zootaxa, 414, 1-66. https://doi.org/10.11646/zootaxa.4146.1.1

Luzzatto, D. \& Pujol, M. G. (2014). Los caballitos de mar. En E. A. Vallarino (Ed.). Cuadernos de educación ambiental. (pp. 29-57). Mar del Plata, Argentina: Universidad Nacional de Mar del Plata.

McNyset, K. M. (2005). Use of ecological niche modeling to predict distributions of freswater fish species in Kansas. Ecol. Freshwater Fish, 14(3), 243255. https://doi.org/10.1111/j.16000633.2005.00101.x

McPherson, J. M. \& Vincent, A. C. J. (2004) Assessing East African trade in seahorse species as a basis for conservation under international controls. Aquat. Conserv., 14(5), 521-538. https://doi.org/10.1002/aqc.629

Pastor-Gutiérrez, L., Piloto-Cubero, Y., Corrada-Wong, R. I. \& Chevalier-Monteagudo, P. P. (2011). Estudio de las poblaciones de caballitos de mar en dos zonas de la costa norte de la Habana y Pinar del Río, Cuba. Rev. Cien. Mar. Cost., 3, 171-181. https://doi. org/10.15359/revmar.3.13

Pearson, R. G., Thuiller, W., Araújo, M. B., Martínez-Meyer, E., Brotons, L., McClean, C., \& Lees, D. C. (2006). Model-based uncertainty in species range prediction. J. Biogeogr., 33(10), 17041711. https://doi.org/10.1111/j.13652699.2006.01460.x

Peterson, A. T. (2001). Predicting species' geographic distributions based on ecological niche modeling. The Condor, 103(3), 599-605. https://doi.org/10.16 50/0010-5422(2001)103[0599:PSGD$\mathrm{BO}] 2.0 . \mathrm{CO} ; 2$ 
Phillips, S. J., Anderson, R. P. \& Schapire, R. E. (2006). Maximum entropy modeling of species geographic distributions. Ecol. Model., 190(3-4), 231-259. https://doi. org/10.1016/j.ecolmodel.2005.03.026

Pimm, S. L., Jenkins, C. N., Abell, R., Brooks, T. M., Gittleman, J. L., Joppa, L. N., \& Sexton, J. O. (2014). The biodiversity of species and their rates of extinction, distribution, and protection. Science, 344(6187), 1246752. https://doi. org/10.1126/science. 1246752

Rosa, I. L., Oliveira, T. P. R., Castro, A. L. C., Souza Moraes, L. E., Xavier, J. H. A., Nottingham, M. C., \& Monteiro-Neto, C. (2007). Population characteristics, space use and habitat associations of the seahorse Hippocampus reidi (Teleostei: Syngnathidae). Neotro. Ichthyology,

5(3), 405-414. https://doi.org/10.1590/ S1679-62252007000300020

SEMARNAT. Secretaria de Medio Ambiente y Recursos Naturales. (2010). NOM-059-SEMARNAT-2010. Norma Oficial Mexicana (NOM-059-SEMARNAT2001), Protección ambiental-Especies nativas de México de flora y fauna silvestres-Categorías de riesgo y especificaciones para su inclusión, exclusión o cambio-Lista de especies en riesgo. Recuperado en abril 17, 2017, disponible en http://dof. gob.mx/nota_detalle_popup.php?codigo $=5173091$

Shelton, A. O., Thorson, J. T., Ward, E. J. \& Feist, B. E. (2014). Spatial semiparametric models improve estimates of species abundance and distribution. Can. J. Fish. Aquat. Sci., 71(11), 1655-1666. https://doi.org/10.1139/cjfas-2013-0508
Soberon, J. \& Peterson, A. T. (2005). Interpretation of Models of Fundamental Ecological Niches and Species' Distributional Areas. Biodivers. Inform., 2, 1-10. https://doi.org/10.17161/ bi.v2i0.4

Teixeira, R. L. \& Musick, J. A. (2001). Reproduction and food habits of the lined seahorse, Hippocampus erectus (Teleostei: Syngnathidae) of Chesapeake Bay, Virginia. J. Bras. Biol., 61(1), 79-90. https://doi.org/10.1590/S003471082001000100011

Toledo, V. M. \& Ordóñez M. J. (2009). Zonas ecológicas de México. Recuperado en abril 11, 2017, disponible en http:// www.conabio.gob.mx/informacion/ metadata/gis/zecol1mgw.xml?_httpcache $=$ yes\&_xsl=/db/metadata $/$ xsl/fgdc_ html.xsl\&_indent $=$ no

Thuiller, W., Lafourcade, B., Engler, R. \& Araújo, M. (2009). Biomod - a plataform for ensamble forecasting of species distributions. Ecography, 32(3), 369-373. https://doi.org/10.1111/ j.1600-0587.2008.05742.x

Valavanis, V. D., Pierce, G. J., Zuur, A. F., Palialexis, A., Saveliev, A., Katara, I. \& Wang, J. J. (2008). Modelling of essential fish habitat based on remote sensing, spatial analysis and GIS. $H y$ drobiologia, 612(1), 5-20. https://doi. org/10.1007/s10750-008-9493-y

Vari, R. P. (1982). Order Gasterosteiformes, Suborder Syngnathoidei (Doryrahmphinae, Syngnathinae, Hippocampinae). In Tee-Van, J. (Ed.), Fishes of the Western North Atlantic (pp. 178-193) New Haven, EE. UU.: Sears Foundation for Marine Research, Yale University.

Villaseñor, J. L. \& Tellez-Valdés, O. (2004). Distribución potencial del género Jefea (Asteracea) en México. Serie Botánica, 75(2), 205-220. 
Vincent, A. C. J., Foster, S. J. \& Koldewey, H. J. (2011). Conservation and management of seahorses and other Syngnathidae. J. Fish Biol., 78(6), 1681-1724. https://doi.org/10.1111/ j.1095-8649.2011.03003.x

Vincent, A. C. J. (1996). The international trade in seahorses. Cambridge, UK.: Traffic International Report.

Wiley, E. O., McNyset, K. M., Peterson, A. T., Robins, C. R. \& Stewart, A. M. (2003). Niche modeling and geographic range predictions in the marine environment using a machine-learning algorithm. Oceanography, 16(3), 120-127. https:// doi.org/10.5670/oceanog.2003.42
Zhang, X. \& Vincent, A. C. J. (2017). Integrating multiple datasets with species distribution models to inform conservation of the poorly-recorded Chinese seahorses. Biol. Cons., 211(A), 161-171. https://doi.org/10.1016/j.biocon.2017.05.020

Zhang, X. \& Vincent, A. C. J. (2018). Predicting distributions, habitat preferences and associated conservation implications for a genus of rare fishes, seahorses (Hippocampus spp.). Divers. Distrib., 24(7), 1005-1017. https://doi. org/10.1111/ddi.12741 
